TENDENCIAS

Revista de la Facultad de Ciencias

Económicas y Administrativas.

Universidad de Nariño ISSN-E 2539-0554

Vol. XXI No. 2 - 2do Semestre 2020,

Julio-Diciembre - Páginas 45-62

\title{
LA INNOVACIÓN EN MARKETING DE LAS EMPRESAS AGROINDUSTRIALES DE LA PROVINCIA DE SUGAMUXI ${ }^{1}$
}

\author{
THE INNOVATION IN MARKETING OF AGRO-INDUSTRIAL COMPANIES IN THE \\ PROVINCE OF SUGAMUXI
}

\section{A INOVAÇÃO EM MARKETING DE EMPRESAS AGRO INDUSTRIAIS NA PROVÍNCIA DE SUGAMUXI}

\author{
HERRERA RODRIGUEZ_Jenny Mairena; OTÁLORA GÓMEZ_Lina María; \\ LOZANO RUEDA_Oscar Mauricio
}

\begin{abstract}
Magíster (C) en Seguridad y Salud en el Trabajo, Universidad Pedagógica y Tecnológica de Colombia. Docente Facultad Ciencias Administrativas y Contables, Universidad de Boyacá. E-mail: jenmaiherrera@uniboyaca.edu.co, Colombia.

Magíster (C) en Neuromarketing, Universidad Internacional de la Rioja. Docente Facultad Ciencias Administrativas y Contables, Universidad de Boyacá. E-mail: 1motalora@uniboyaca.edu.co, Colombia.

Magíster (C) en Dirección y Gestión de Recursos Humanos, Universidad Internacional de la Rioja. Docente Facultad Ciencias Administrativas y Contables, Universidad de Boyacá. E-mail: omlozano@uniboyaca.edu.co, Colombia.
\end{abstract}

Recibido: 29 de abril de 2020

Aprobado: 24 de agosto de 2020

DOI: https://doi.org/10.22267/rtend.202102.140

\footnotetext{
${ }^{1}$ Este artículo es resultado de las labores en el Grupo de Investigaciones Sectoriales empresariales y Desarrollo Económico de la Universidad de Boyacá. Los hallazgos, interpretaciones y conclusiones pertenecen a los autores de la investigación titulada Propuesta para la Implementación de un Modelo Gestión de Innovación para las Mipymes Sector Agroindustrial Provincia Sugamuxi.
} 


\title{
RESUMEN
}

El objetivo de este trabajo consiste en diagnosticar la innovación en marketing de las empresas agroindustriales de la Provincia de Sugamuxi para poder evidenciar las falencias y fortalezas, con el fin de crear estrategias que aporten en la competitividad de las compañías. Este análisis se realizó a través de un enfoque cuantitativo de tipo descriptivo y transversal porque se aplicó en una sola ocasión una encuesta a 39 empresas agroindustriales de la Provincia de Sugamuxi. Se determina que año tras año vendan los mismos productos, al mismo cliente con un idéntico empaque y distribución afectando el crecimiento y rentabilidad de la empresa porque no se aumenta la participación del mercado ni nuevos clientes.

Palabras clave: innovación; mercadeo; agroindustria; gestión de innovación; actividad económica regional.

JEL: E31; M31; Q13; O32; R11

\begin{abstract}
The objective of this work is to diagnose the innovation in marketing of the agricultural industry companies of the Province of Sugamuxi in order to demonstrate the shortcomings and strengths in order to create strategies that contribute to the competitiveness of the companies. This analysis was carried out through a quantitative, descriptive and cross-sectional approach, for which a survey was applied to 39 companies on one occasion. of the agricultural industry of the Sugamuxi Province. Its determined achieving that year after year they sell the same products, to the same customer with the same packaging and distribution, affecting the growth and profitability of the company because market share and new customers are not increased.
\end{abstract}

Keywords: innovation; marketing; agricultural industry; management of technological innovation; regional economic activity.

JEL: E31; M31; Q13; O32; R11

\section{RESUMO}

O objetivo deste trabalho é diagnosticar a inovação em marketing das empresas agroindustriais da província de Sugamuxi, a fim de demonstrar as deficiências e os pontos fortes, a fim de criar 
estratégias que contribuam para a competitividade das empresas. Essa análise foi realizada por meio de abordagem quantitativa, descritiva e transversal, para a qual uma pesquisa foi aplicada a 39 empresas em uma ocasião. agroindustriais da província de Sugamuxi. Esta determinado não desenvolvem inovação de marketing, alcançando isso ano após ano, eles vendem os mesmos produtos, para o mesmo cliente, com a mesma embalagem e distribuição, afetando o crescimento e a lucratividade da empresa, porque a participação de mercado e novos clientes não aumentam.

Palavras-chave: inovação; marketing; agroindustriais; gestão da inovação tecnológica; atividade econômica regional.

JEL: E31; M31; Q13; O32; R11

\section{INTRODUCCIÓN}

En un ambiente de negocios tan dinámico como el que se vive hoy en día, las estrategias y políticas implementadas por las empresas, deben estar dirigidas a incrementar su capacidad para la innovación, es decir, el aprovechamiento de los conocimientos y habilidades para generar al interior de la empresa el desarrollo de las capacidades del personal en general, en lo que respecta a la capitalización de nuevas ideas, para mejorar la eficiencia, la productividad y por consiguiente, la competitividad.

Teniendo presente la competitividad e innovación como un factor fundamental en las empresas, se evidencia que países como Colombia presentan dificultades en estos factores; en especial el sector agrícola, donde el PIB agrícola alcanzó un promedio a nivel nacional del 2\% y el departamento de Boyacá tan solo presentó 0,5\% en el año 2017 (Cámara de Comercio de Sogamoso, 2016). De igual manera el Índice de Competitividad y de Innovación evidencia que el Departamento presenta una situación negativa, en especial el sector agroindustrial que presenta baja competitividad, nivel tecnológico mínimo que genera ineficiencia en las organizaciones (Consejo Privado de Competitividad, 2017, p. 54).

Para promover esta competitividad se debe evidenciar algunos factores importantes en especial en la Provincia de Sugamuxi que hace parte del Departamento de Boyacá, de los cuales se mencionan principalmente la "innovación, conocimiento, tecnología, mercadeo y talento humano" (Becerra y Cruz, 2014, p. 112). Con estos ítems, el presente artículo tiene el enfoque de unificar dos factores: la 
innovación y el mercadeo como estrategia de competitividad para poder diagnosticar a las empresas agroindustriales en la innovación en marketing y poder generar una propuesta que aporte a las organizaciones y genere competitividad en el sector.

Se habla de la innovación en marketing porque se ha convertido sobre todo en la última década, en un proceso de absoluta necesidad para aquellas empresas que buscan no solo un buen posicionamiento dentro del mercado al que pertenecen, sino la perdurabilidad en el tiempo; dicho esto se requiere involucrar en ella el concepto creatividad que ligado a la innovación y el marketing consolidan una verdadera posibilidad de establecer ventajas competitivas (Kirkberg, 2013).

Aunque diferentes autores han destacado la innovación, marketing y desarrollo de redes como los principales desafíos en el caso de la agroindustria, aún no se han encontrado estudios que se refieran específicamente a innovaciones de marketing; considerando que existen para innovaciones de productos y procesos, especialmente en los alimentos industria (Alston, 2010; Avermaete et al., 2003; Capitanio et al., 2010; Earle, 1997; Fritz y Schiefer, 2008; Furtan y Sauer, 2008; Grunert et al., 2008; Suwannaporn y Speece, 2003; Traill y Meulenberg, 2002), lo cual es un desafío como investigadores el generar estudios y aportes en este factor.

Por lo anterior, el desarrollo del artículo se presenta en cuatro partes: en el primero, se abordan los aspectos teóricos relacionados con la innovación en marketing, modelo de innovación y agroindustria; en la segunda parte, se presenta la metodología utilizada en la investigación; en la tercera los resultados y por último las conclusiones.

\section{REFERENTES TEÓRICOS}

Hoy en día, las empresas deben estar en una búsqueda constante de aspectos que garanticen estar a la vanguardia del mercado y por tanto permitan la supervivencia. En ese sentido la innovación es un tema que reviste de gran importancia, a continuación, se presenta un acercamiento a la revisión bibliográfica realizada: 


\section{Innovación}

Realmente son numerosas las definiciones sobre innovación, esto debido a los cambios de pensamiento propios de los diferentes escenarios de la economía; Morales, Ortíz, y Arias (2012) mencionan que, tradicionalmente la innovación es entendida como la creación o modificación de un producto para su posterior introducción al mercado, idea que acerca al empresario a entender que la innovación no se limita a aquellas modificaciones realizadas en el producto o servicio, sino que su significado se extiende a la respuesta que la parte demandante otorgue a nuestra propuesta de innovación, poniendo a consideración si esta tubo o no un impacto. Por otra parte, no se puede desligar el hecho de que la innovación es un proceso basado en el conocimiento existente sobre el producto o servicio en cuestión; Illera (1977), manifiesta que la innovación puede ser el resultado de una investigación realizada, pero no se puede descartar el hecho de que esta puede también ser el producto de un accidente o descubrimiento imprevisto, de esta forma la innovación es propuesta como un proceso flexible entre la investigación realizada y los aportes del entorno en cual esta se desarrolle.

Una política de innovación en forma adecuada y oportuna debe considerarse como una estrategia para enfrentar la competencia, ganar nuevos mercados e incrementar sus índices de productividad. Por lo tanto, una actitud mental positiva hacia la innovación por parte de la gerencia de marketing como estrategia vital de mercado, conduce inexorablemente a la organización a definir y capitalizar otras ventajas comparativas y, por ende, a lograr una consolidación en el mercado y exhibir mayores niveles de competitividad (Márquez, 2003. p. 79).

La innovación más habitual en estructuras empresariales pequeñas y medianas está relacionada con el marketing, por su tamaño y facilidad de crear estrategias. Lo que refleja la necesidad de buscar sinergias y colaboraciones entre ambas como fórmula de desarrollo organizativo.

\section{Marketing}

Al hablar de mercadeo se deben considerar los factores externos de la organización que conllevan al reconocimiento de las necesidades del mercado (Sixto, 2014); Kotler y Armstrong (2007) plantean que el marketing es un proceso social y administrativo del cual se espera que tanto las organizaciones 
como los individuos, obtienen lo que desean al crear e intercambiar valor con otros, esto incluye el establecimiento de relaciones redituables, con valor agregado, con los clientes.

Según Estrada et al. (2015). "el marketing va dirigido principalmente a los clientes, y tiene como finalidad alcanzar los objetivos establecidos por la organización, logrando así satisfacer al consumidor" (p. 272), por ende, se considera una actividad planteada en pro del crecimiento de la empresa, pero enfocada a la identificación y satisfacción de las necesidades del cliente; en la actualidad el concepto de marketing ha evolucionado hasta alcanzar una madurez acorde a las necesidades de las organizaciones, se podría decir incluso que el marketing se ha hecho adulto, porque ha incorporado las herramientas de análisis estratégico que han surgido hace algunas décadas pero que ahora se representan con mayor fuerza a partir de la presencia del marketing estratégico y sus derivados como el marketing segmentado, la estrategia de posicionamiento, y el marketing relacional (De Vicuña Ancín, 2016, p 39). La innovación y acciones de marketing relacional, ya sea de manera directa o indirecta, deberían ser consideradas como pilares básicos de la estrategia marketing a fin de alcanzar el éxito empresarial. Reiterar que dichas conclusiones deben entenderse en el marco de la investigación realizada (Küster y Vila, 2010. p. 201).

\section{Innovación en marketing}

La innovación es un componente ligado a las empresas en las condiciones actuales del mercado,

Kirkberg (2013, p. 64) comenta que creatividad, innovación y marketing son conceptos que normalmente llegan a ser analizados por separado; sin embargo, estos son factores claves para alcanzar el éxito en el proceso de creación, desarrollo y lanzamiento de nuevos productos y servicios al mercado, tanto en empresas ya existentes como en aquellas que esperan entrar por primera vez a un mercado, por lo que definitivamente deben estar integrados; entendido de esta manera, la innovación debe ser analizada como parte de un conjunto en el que la creatividad y el marketing aportan factores importantes para su buen desarrollo. Ramos, Polo, Arrieta y Vega (2018) sugieren que la innovación en mercadotecnia es un proceso asociado a la generación de ventajas competitivas orientadas a las cimentación de barreras de acceso para la competencia, toda vez que los cambios generados en la promoción, precio, diseño y posicionamiento del producto o servicio, se convierten en aspectos novedosos generadores de éxito siempre y cuando estos representen una diferencia importante en la variable tiempo, de tal forma que las empresas competidoras al intentar reproducir o adquirir aquellos 
recursos que se han utilizado para la innovación, se encuentren en una posición de desventaja frente al tiempo que la organización ya está empleando para generar nuevas innovaciones.

Una innovación en marketing es la puesta en marcha de nuevas técnicas, métodos o planes que impliquen nuevas o mejoras significativas de: estrategias, posicionamiento o segmentación; de comercialización, distribución o venta; de comunicación, promoción o publicidad; de políticas de fijación de precio; o de diseño de productos o de envases.

\section{Modelo de gestión para el proceso de la innovación en marketing}

Dentro de la presente investigación se realizaron estudios sobre modelos de innovación que aportan al área de marketing, descubriendo que los modelos se basan no solo en un área sino en varias dado que la empresa debe generar la innovación en diferentes factores para generar una competitividad que sea difícil de imitar.

El modelo seleccionado es un modelo de gestión en innovación que está basado principalmente en la innovación de marketing, innovación de productos, innovación de procesos, innovación de redes, desarrollo de recursos humanos en innovación, innovación administrativa, innovación estratégica e innovación de visión y política, que permite cubrir los factores principales de un sistema de negocios con el fin de alcanzar el rendimiento real de innovación en una organización (Maier, et al., 2013, p. 732).

Como se presenta en la Figura 1, este modelo logra identificar el grado de innovación en cualquier parte de la organización, el ambiente propicio que debe tener la innovación y la valoración por diferentes áreas sobre una idea con el fin de identificar las fortalezas y debilidades y aumentar el valor de la misma (Maier et al., 2013, p. 731). 


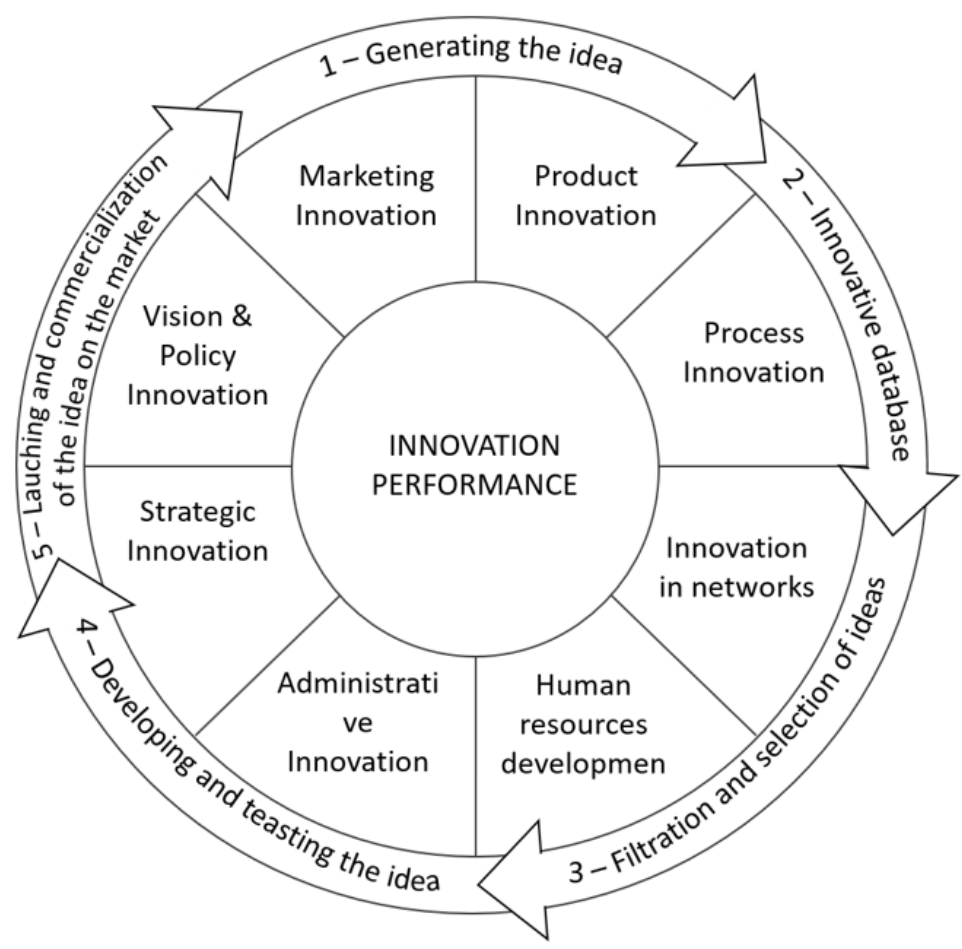

Figura 1. Modelo de gestión para el proceso de innovación.

Fuente: Tomado de Maier et al., (2013). Achieving performance of organization by developing a model of innovation management. In European Conference on Innovation and Entrepreneurship (Vol. 2, p. 732). Academic Conferences International Limited.

Consecuentemente, este modelo utiliza el método QFD (Despliegue de la función de calidad), con el fin de mejorar la satisfacción de los clientes e integrar los requisitos de innovación en el área de marketing.

El modelo selecciona unos requisitos que se deben cumplir para evidenciar la innovación en la empresa, dentro de ellos se seleccionaron el aumento de mercado, crecimiento de nuevos productos y crecimiento en la rentabilidad comercial. Estos requisitos son medidos por medio de unos indicadores que calculan el desempeño de la innovación a nivel de la organización (Maier et al., 2013, p. 732). Los indicadores para la innovación en marketing son:

- La cantidad de cambios significativos realizados en el diseño y empaque de los productos

- La cantidad de nuevos clientes 
- Número de innovaciones para aumentar la cuota de mercado

- El porcentaje de aumento en los beneficios como resultado de las innovaciones en marketing

- La innovación en la rentabilidad de comercialización (ganancia / capital invertido obtenido) (Maier et al., 2013, p. 735).

\section{Agroindustrial}

Se define la agroindustria como "una empresa que elabora materias primas agrícolas, donde el grado de elaboración puede ir desde la obtención de la materia prima hasta un proceso de transformación de la misma" (James Austin, citado en Martínez et al., 2014, p. 16), esto comprende mejoramiento del producto (característica, apariencia, atributos), empaque y distribución.

Adicionalmente, la agroindustria se clasifica por un nivel cero que abarca solo el producto agrícola y no tienen ningún cambio es decir se conserva tal cual es; un nivel uno que se da cuando el producto tiene una etapa primaria de transformación por ejemplo en pulpa de fruta; o un nivel dos donde el producto ya tiene una transformación más avanzada a llegar a un enlatado, polvo, conserva, etc (Isidro Planella, citado en Martínez et al., 2014, p.16).

\section{METODOLOGÍA}

La investigación es de tipo descriptivo porque realiza el diagnóstico de la innovación en marketing de las Mipymes agroindustriales de la Provincia de Sugamuxi. Su diseño es transversal con un enfoque cuantitativo ya que se aplicará un instrumento en una sola ocasión, para lograr a través de la obtención de datos realizar el diagnóstico y la caracterización de los factores más relevantes en estas organizaciones.

El cuestionario que se utilizó es el instrumento del modelo de gestión para el proceso de innovación, según los indicadores de medición del rendimiento en innovación (Maier et al., 2013, p. 732), puesto que este abarca los diferentes aspectos que se pueden llegar a tener encuentra en una organización.

Así mismo el instrumento se aplicó a los gerentes de las empresas agroindustriales, que es una población finita, ya que se estableció un tamaño de 76 empresas agroindustriales de la provincia de Sugamuxi obtenida del directorio de la Gobernación de Boyacá y de la base de datos de la Cámara de 
Comercio de Sogamoso 2018, de las cuales se verificaron que fueran mipymes agroindustriales y por lo tanto resultaron 39 empresas que en consecuencia todas aceptaron la aplicación del instrumento.

\section{RESULTADOS}

Teniendo presente los cinco indicadores de innovación en marketing del modelo estudiado, los cuales son:

- Cantidad de cambios significativos realizados en el diseño y empaque de los productos

- Cantidad de nuevos clientes

- Número de innovaciones para aumentar la cuota de mercado

- El porcentaje de aumento en los beneficios como resultado de las innovaciones en marketing

- La innovación en la rentabilidad de comercialización (ganancia / capital invertido obtenido) (Maier et al., 2013, p. 735).

Se presenta a continuación un análisis de las 39 empresas agroindustriales de la Provincia de Sugamuxi con respecto a su desempeño en innovación en marketing:

- En cuanto a los cambios significativos que se han realizado en el diseño y empaque de los productos durante los últimos tres años se presenta (Figura 2):

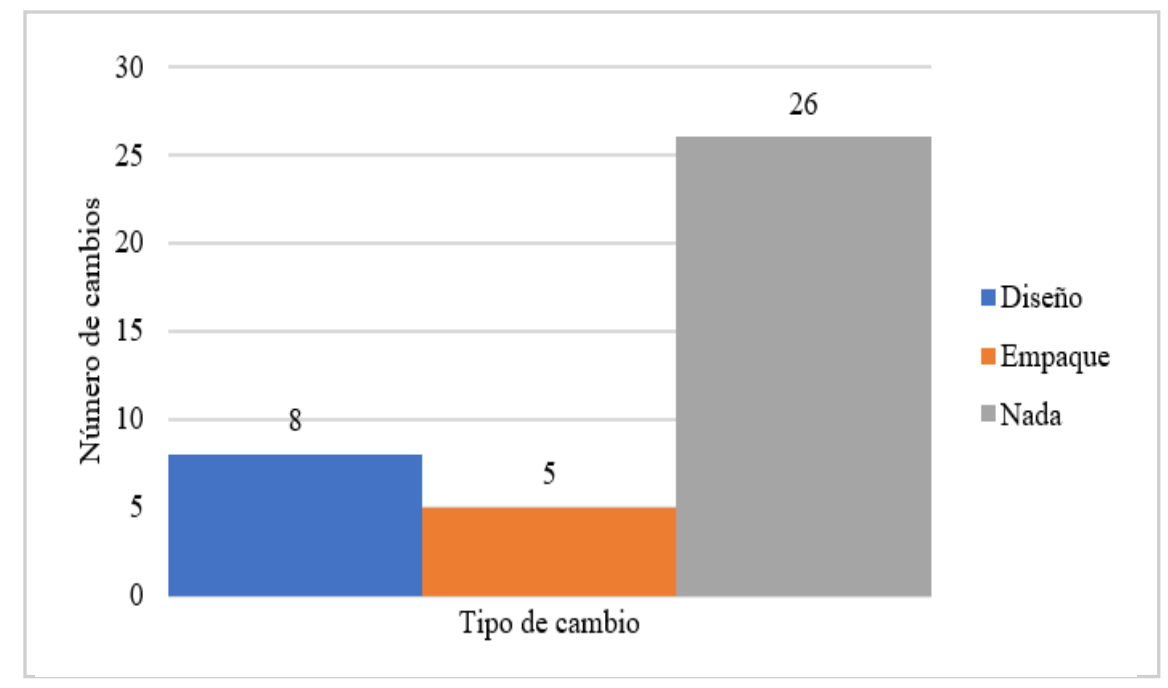

Figura 2. Tipo de cambios.

Fuente: datos de la encuesta. 
Solo 13 empresas afirmaron hacer algún cambio significativo en los últimos tres años respecto al diseño o empaque. De las cuales 8 empresas son en diseño y 5 empresas en empaque lo que evidencia una ineficiencia en este indicador y además que en Boyacá las empresas agroindustriales aún siguen siendo artesanales en la forma de producción, pues en su gran mayoría desconocen técnicas administrativas debido también a su formación académica.

La Figura 2 buscaba conocer los cambios que las empresas agroindustriales han adoptado en sus productos, lo cual resulta negativo para el sector agroindustrial ya que la mayoría asegura no haber realizado ningún cambio en diseño o empaque, en la Figura 3, se observa que una de las 39 empresas afirma haber realizado 7 cambios en los últimos 3 años, seguida de una empresa que ha realizado 3. Los autores Hartwich y Negro (2010, p. 1) desarrollaron un estudio en Nueva Zelanda también en el sector agroindustrial, concluyendo de igual forma que las innovaciones de marketing en cuanto a envase y embalaje es negativo, puesto que no existe relaciones de cooperación y la mayoría de estos empresarios son informales; para este caso de Sugamuxi el análisis que se puede hacer es que estos empresarios son empíricos y necesitan desarrollar actividades que permitan mejorar su posicionamiento y competitividad. Así mismo otras investigaciones de Schmidt y Rammer (2007, p. 6); Mothe y Nguyen (2012) donde aplicaron un instrumento que contenía una pregunta muy similar, aseguraron que el cuestionamiento responde a las innovaciones en marketing que una organización puede desarrollar.

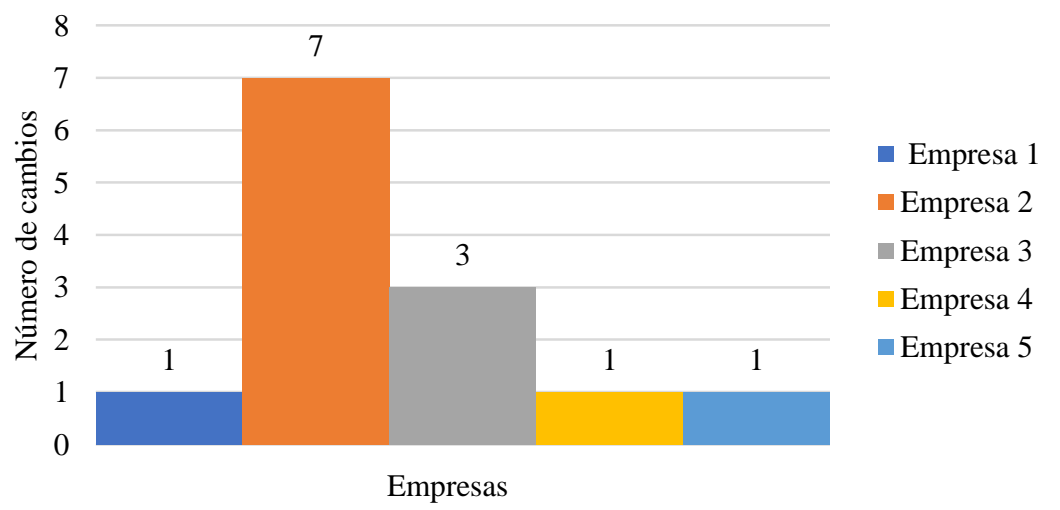

Figura 3. Número de cambios

Fuente: resultados de la encuesta. 
- En relación a la cantidad de nuevos clientes que ha adquirido la empresa durante los últimos tres años observamos (Figura 4):

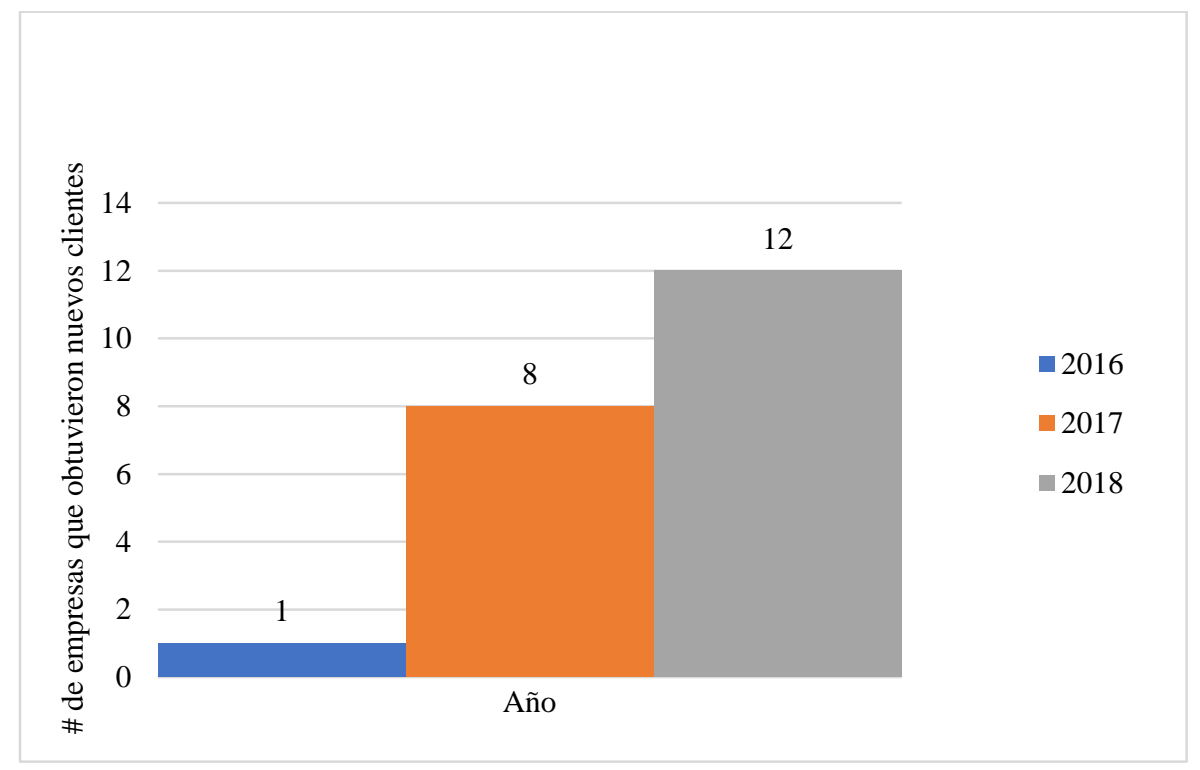

Figura 4. Nuevos clientes obtenidos en los últimos tres años.

Fuente: datos de la encuesta.

Para el año 2016 solo una empresa aseguró haber obtenido nuevos clientes, pero para los años siguientes la cifra aumentó, de esta forma se encuentra que ocho empresas lograron conseguir nuevos clientes en el año 2017 y finalmente para el 2018 fueron doce las que lograron esta meta. Aunque en el instrumento no se indaga sobre el porqué del aumento de clientes a través de los años, podría asumirse que se debe a la demando de los productos derivados de la agroindustria.

En la Figura 5 se observa que las 8 empresas consideradas, incrementaron en 65 nuevos clientes, comportamiento que es bastante diferenciado entre las diferentes empresas. Mientras en la cuarta empresa considerada, se alcanzaron 37 clientes. En las otras siete empresas, el crecimiento fue de un total de 28 nuevos clientes. Entonces, de 65 nuevos clientes de las 8 empresas consideradas, una sola obtuvo 37 nuevos clientes, que representan el 55,22\% del total y las siete empresas restantes, obtuvieron el $44.78 \%$ del incremento total de nuevos clientes. A pesar de pertenecer las empresas a una misma industria, todas tiene cambios diferentes, esto permite apreciar y entender que cada empresa es única y está determinada por las actividades que encamine para ser más competitivo. 
La innovación en marketing de las empresas agroindustriales de la provincia de Sugamuxi Herrera Rodriguez_ Jenny Mairena; Otálora Gómez_Lina María; Lozano Rueda_ Oscar Mauricio

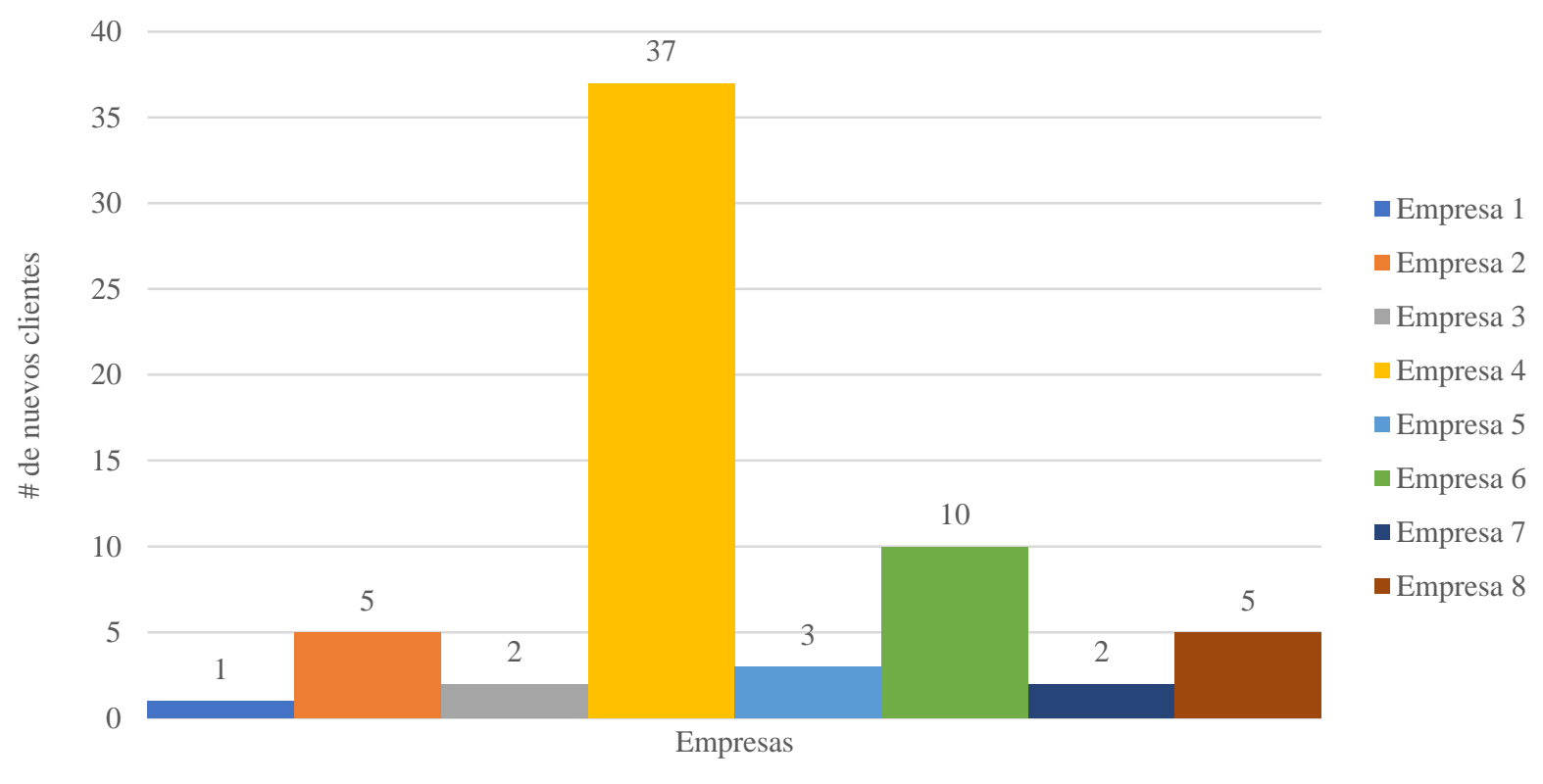

Figura 5. Nuevos clientes obtenidos por las empresas en el año 2017.

Fuente: datos de la encuesta.

En la Figura 6, se puede ver un aumento en el número de clientes respecto a años anteriores, lo que supondría que las empresas han venido mejorando en el tiempo, pues son más las empresas y mayor cantidad de clientes.

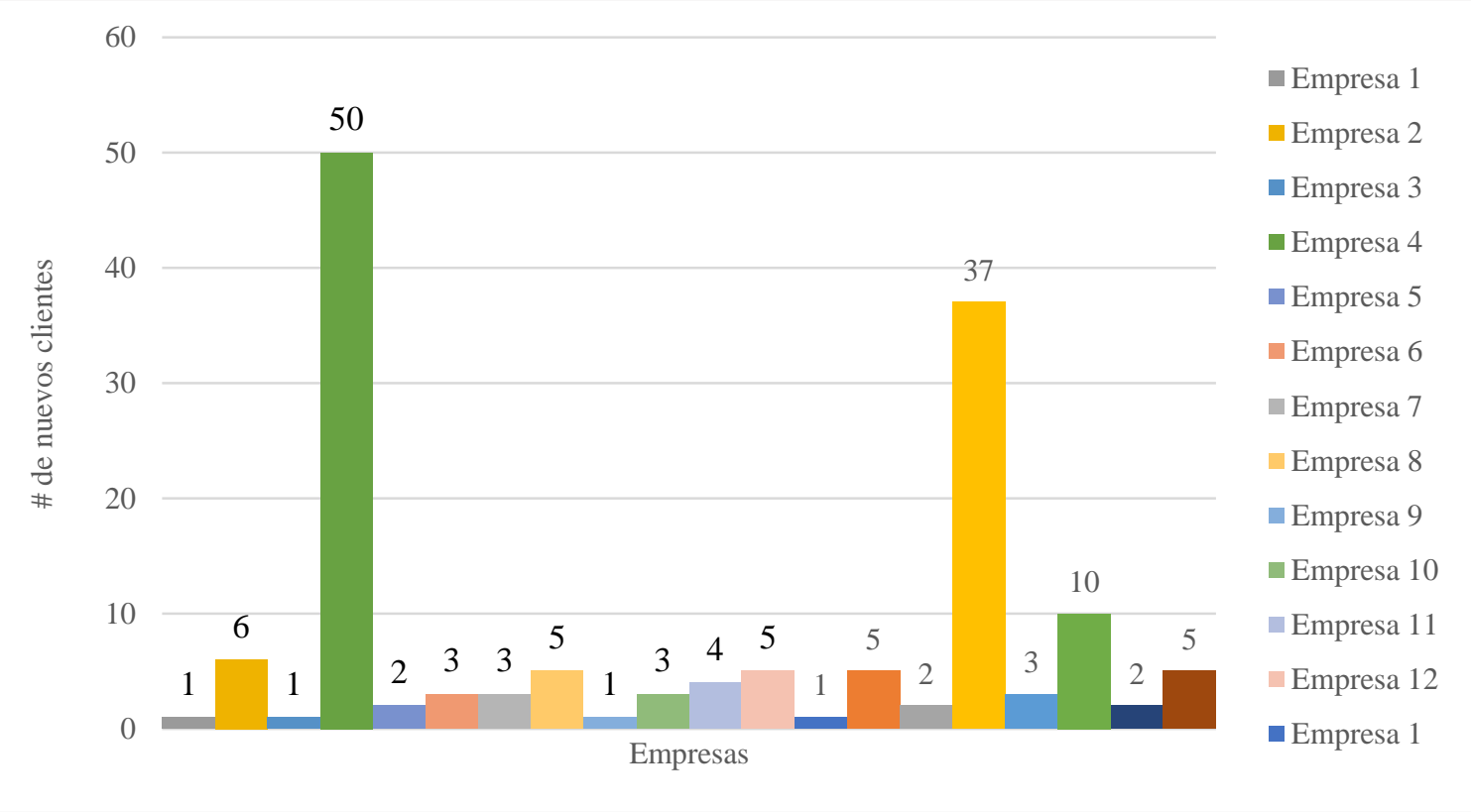

Figura 6. Nuevos clientes obtenidos por las empresas en el año 2017.

Fuente: datos de la encuesta. 
- Del tercer y cuarto indicador que es el número de innovaciones que se han realizado para aumentar la participación de la empresa en el mercado y que consideran estimado como beneficio, durante los últimos tres años se presenta (Figura 7):

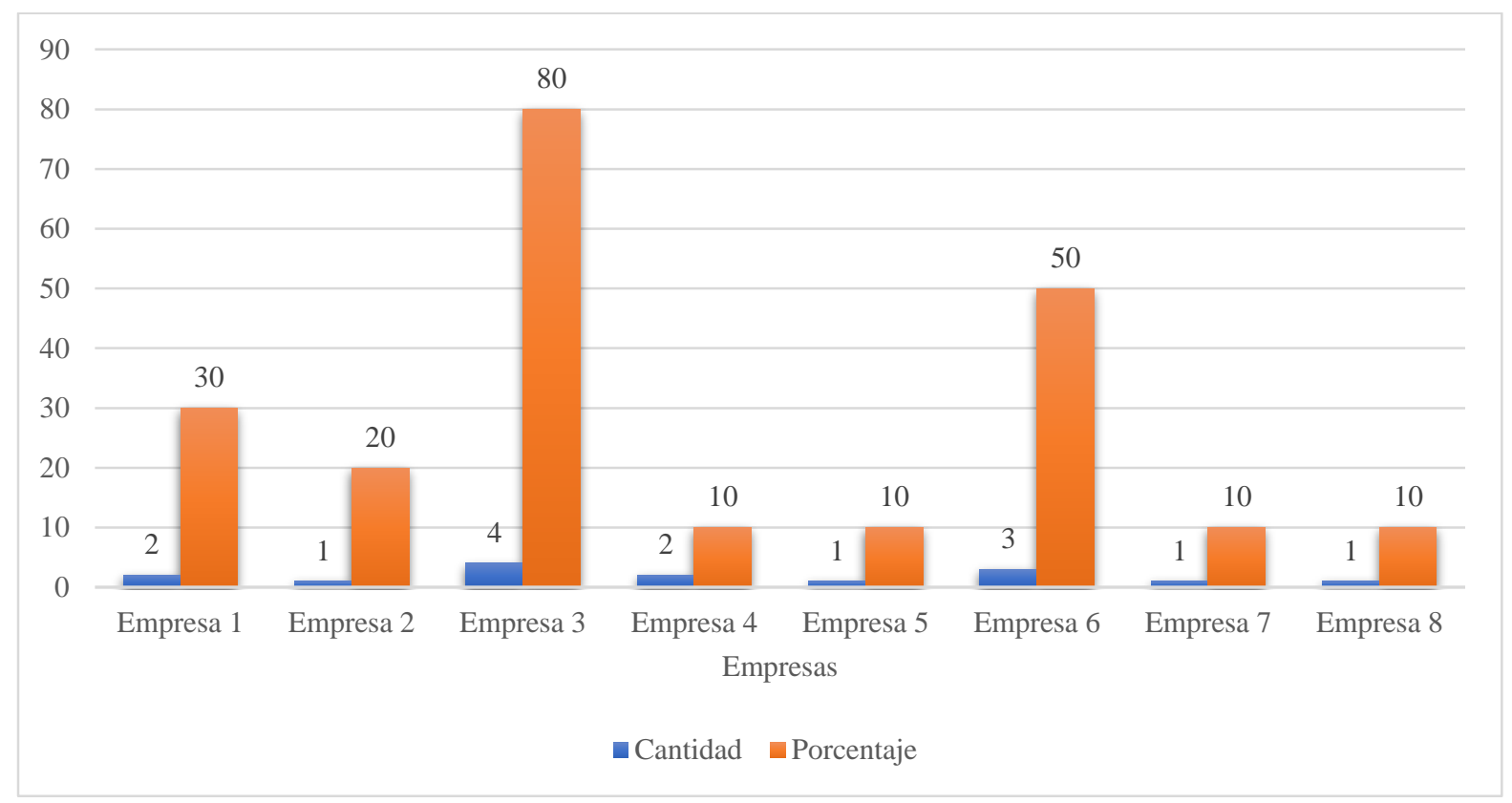

Figura 7. Cantidad de Innovaciones e Incremento de participación en el mercado.

Fuente: datos de la encuesta.

Una visión en conjunto del estudio, permite encontrar que la cuarta empresa, considerada la del mayor aumento de clientes, hizo 2 innovaciones y su participación en el mercado solamente aumentó en el $10 \%$, en tanto que las empresas que más innovaron, mostraron un incremento mucho mayor de su participación en el mercado, sin que esto muestre una relación directa con la consecución de nuevos clientes. Podría asumirse que la participación porcentual en el mercado para estas empresas está representada en el impacto que sus innovaciones tienen en sus clientes actuales y no en la obtención de nuevos compradores.

Logrando así mismo que las empresas que generaron una innovación, tengan mayor participación de mercado con relación a los clientes actuales lo que con lleva aumentar el quinto indicador que es la rentabilidad de comercialización puesto que al invertir en innovación creció la demanda del cliente por los productos generando un incremento en las ventas. 
Lo anterior, es referente a las 8 empresas de las 36, es decir que el $22 \%$ de las empresas agroindustriales de la Provincia de Sugamuxi generan algo de innovación en marketing lo que ha aportado en los últimos años al crecimiento de las empresas.

\section{CONCLUSIONES}

En el transcurso de la investigación se ha logrado evidenciar el impacto económico, ambiental y social del sector agroindustrial en el departamento de Boyacá, por cuanto cada vez reviste de más importancia el desarrollo de una investigación que apunta a fortalecer los procesos y actores que la intervienen.

Se recomienda que la agroindustria en la ciudad de Sogamoso trabaje de manera más unida, pues generando asociatividad entre el sector podrían generarse estrategias enfocadas a competir en mercados globalizados y no tanto en mercados regionales, es decir, abarcar mercados mucho más grandes que favorezcan el crecimiento de la región, se hace indispensable el trabajo entre empresa, academia y estado para unir fuerzas y mejorar el trabajo.

Como se encuentra en la revisión de literatura realizada, la generación de nuevas ideas contribuye a las organizaciones a la generación de decisiones estratégicas que permitan la supervivencia de las empresas.

El concepto de innovación se ha convertido en un espacio común en ámbitos muy diversos de la actividad humana. La complejidad de los procesos de innovación y el entorno en el que se desarrollan generan la necesidad de modelos de comprensión eficientes y replicables.

La aplicación del instrumento ha permitido evidenciar que las empresas agroindustriales han quedado un poco atrasadas en aspectos de innovación en marketing, por lo que se hace necesario una intervención por parte de la academia hacia los empresarios, contribuyendo como primera medida a la divulgación de resultados y, en segunda medida, a la generación de estrategias que busquen concientizar de la importancia de generar nuevas ideas que propendan por el éxito organizacional. 
El mundo avanza, las necesidades cambian, los deseos cada vez son más ambiciosos, por cuanto las empresas deben garantizar que sus productos también sean acordes a dichos cambios. Se hace necesario que las empresas tengan como premisa la necesidad del cliente y del mercado, para que cada cambio implementado en el empaque, diseño o producto sea diferenciador.

Las empresas no pueden esperar que los clientes aumenten si no ofertan productos innovadores, este estudio en las organizaciones agroindustriales permite evidenciar la falta de innovación en aspectos del producto, como academia se puede contribuir en la actualización de aspectos relacionados al marketing, formando a empresarios idóneos y con habilidades de generar estrategias acordes al momento que vivimos.

Además, realizar un análisis solido al consumidor a fin de reunir la información real respecto a sus necesidades, deseos, características que conduzcan a crear estrategias y desarrollar ideas innovadoras para cumplir sus expectativas en cuanto al producto, servicio, empaque, distribución, comunicación, relaciones.

\section{REFERENCIAS}

(1) Alston, J. (2010). The benefits from agricultural research and development, innovation, and productivity growth. OECD Food, Agriculture and Fisheries, Working Papers, No. 31, OECD Publishing, Paris. doi: $10.1787 / 5 \mathrm{~km} 91 \mathrm{nfsnkwg-en}$

(2) Avermaete, T., Viaene, J., Morgan, E. y Crawford, N. (2003). Determinants of innovation in small foods firms. European Journal of Innovation, 6(1), 8-17. doi: 10.1108/14601060310459163

(3) Becerra, C. y Cruz, J. (2014). Diagnóstico de la competitividad agroindustrial en el departamento de Boyacá para el año 2011. Revista de Investigación, Desarrollo e Innovación, 4(2), 111-123. doi: $10.19053 / 20278306.2961$

(4) Cámara de Comercio de Sogamoso. (2016). Estudio de percepción económica del municipio de Sogamoso 2016. Recuperado de http://camarasogamoso.org/wp-content/uploads/2017/01/EST_ECONOMICO.pdf

(5) Capitanio, F., Coppola, A. y Pascucci, S. (2010). Product and process innovation in the Italian food industry. Agribusiness, 26(4), 503-518. doi: 10.1002/agr.20239 
(6) Consejo Privado de Competitividad. (2017). Índice de Competitividad Departamental. Recuperado de https://compite.com.co/proyecto/indice-departamental-de-competitividad-

2016/\#: :text=El\%20\%C3\%8Dndice\%20Departamental\%20de\%20Competitividad\%202017\%2C\%20de \%201a\%20misma\%20manera,y\%20iii)\%20sofisticaci\%C3\%B3n\%20e\%20innovaci\%C3\%B3n.

(7) De Vicuña Ancín, J. M. S. (2016). El plan de marketing en la PYME. Esic Editorial.

(8) Earle, M. (1997). Innovation in the food industry. Trends in Food Science \& Technology, 8(5), 166-175. doi: 10.1016/S0924-2244(97)01026-1

(9) Estrada, H., Iglesias, M. A., Saumett, H., Osorio, C., Rosero, K., Uribe, A. y Sánchez, M. (2015). Gestión de marketing para el sector Agroindustrial. Barranquilla, Colombia: Universidad Simón Bolívar.

(10) Fritz, M. y Schiefer, G. (2008). Innovation and system dynamics in food networks. Agribusiness, 24(3), 301-305. doi: 10.1002/agr.20170

(11) Furtan, W. y Sauer, J. (2008). Determinants of food industry performance: survey data and regressions for Denmark. Journal of Agricultural Economics, 59(3), 555-573. doi: 10.1111/j.1477-9552.2008.00164.x

(12) Gobernación de Boyacá. (2016). Plan de Desarrollo Boyacá 2016-2019 Creemos en Boyacá, tierra de paz y libertad. Recuperado de https://www.boyaca.gov.co/pdd-boyaca-2016-2019/

(13) Grunert, K. G., Jensen, B. B., Sonne, A. M., Brunsø, K., Byrne, D. V., Clausen, C.,... Lettl, C. (2008). User-oriented innovation in the food sector: relevant streams of research and an agenda for future work. Trends in Food Science \& Technology, 19(11), 590-602. doi: 10.1016/j.tifs.2008.03.008

(14) Hartwich, F. y Negro, C. (2010). The role of collaborative partnerships in industry innovation: lessons from New Zealand's dairy sector. Agribusiness, 26(3), 425-449. doi:10.1002/agr.20231

(15) Illera, L. E. (1977). Innovación y Crecimiento Económico. Revista de la Universidad de La Salle, 1(2), 21-28.

(16) Kirkberg, A. S. (2013). Creatividad, Innovación y Marketing: Claves del Éxito Emprendedor. Caderno Profissional de Marketing-UNIMEP, 1(1), 64-74.

(17) Kotler, P. y Armstrong, G. (2007). Marketing. Versión para Latinoamérica. México: PEARSON. Educación.

(18) Küster, I., y Vila, N. (2010). La orientación al mercado y el marketing relacional: efectos en la innovación y el éxito del textil español. Ensayos de Economía, 20(36), 165-202. Recuperado de https://revistas.unal.edu.co/index.php/ede/article/view/27874

(19) Maier, A., Olaru, M., Maier, D. y Marinescu, M. (2013). Achieving performance of organization by developing a model of innovation management. In European Conference on Innovation and Entrepreneurship (Vol. 2, p. 731). Academic Conferences International Limited.

(20) Márquez, O. G. (2003). La innovación como estrategia de marketing. CICAG: Revista del Centro de Investigación de Ciencias Administrativas y Gerenciales, 2(2), 72-81. 
(21) Martínez, L., Sánchez, N. y Sanabria, L. (2014). Caracterización de las Mipymes del sector agroindustrial, en el marco de la aliza estratégica interinstitucional del conocimiento para la competitividad del municipio de Sogamoso Boyacá (Tesis de pregrado). Universidad Pedagógica y Tecnológica de Colombia, Sogamoso.

(22) Morales, M., Ortíz, C. y Arias, M. (2012). Factores determinantes de los procesos de innovación: una mirada a la situación en Latinoamérica. Revista EAN, (72), 148-163.

(23) Mothe, C. y Nguyen, T. (2012). Non-technological and technological innovations: do services differs from manufacturing? An empirical analysis of Luxemburg firms. International Journal of Technology Management, 57(4), 227-244.

(24) Ramos, J., Polo, J., Arrieta, A. y Vega, J. (2018). Impacto de la innovación en marketing sobre la conducta exportadora de las empresas del sector agroindustrial español. Revista De Métodos Cuantitativos Para La Economía $\quad y \quad$ la Empresa, 25, 54-71. Recuperado de https://www.upo.es/revistas/index.php/RevMetCuant/article/view/2310

(25) Schmidt, T. y Rammer, C. (2007). Non technological and technologial innovation: strange bedfellows? ZEW Discussion Paper No. 07-052, ZEW, Mannhein, Germany.

(26) Sixto, J. (2014). Marketing hasta la última definición de la AMA (American Marketing Association). Revista de la Asociación Española de Investigación de la Comunicación, 1(2), 124-132.

(27) Suwannaporn, P. y Speece, M. (2003). Marketing research and new product development success in Thai food processing. Agribusiness, 19(2), 169-188. doi: 10.1002/agr.10047

(28) Traill, W. B. y Meulenberg, M. (2002). Innovation in the food industry. Agribusiness, 18(1), 1-21. doi: 10.1002/agr.10002

Cómo citar este artículo: Herrera, J., Otálora, L. y Lozano, R. (2020). La innovación en marketing de las empresas agroindustriales de la Provincia de Sugamuxi. Tendencias, 21(2), 45-62. https://doi.org/10.22267/rtend.202102.140 